\title{
Long-Term Functionality Prediction for First Time Ischemic Middle Cerebral Artery Stroke Patients Receiving Conventional Medical Treatment
}

\author{
Ju-Lan Yang ${ }^{1,2}$, Chih-Ming Lin $\mathbb{D}^{3-5}$, Ying-Lin Hsu ${ }^{1,6}$
}

'Department of Applied Mathematics, National Chung Hsing University, Taichung, Taiwan; ${ }^{2}$ Department of Rehabilitation Medicine, Changhua Christian Hospital, Changhua City, Taiwan; ${ }^{3}$ Graduate Institute of Statistics and Information Science, National Changhua University of Education, Changhua City, Taiwan; ${ }^{4}$ Department of Neurology, Changhua Christian Hospital, Changhua City, Taiwan; ${ }^{5}$ Department of Post-Baccalaureate Medicine, College of Medicine, National Chung Hsing University, Taichung, Taiwan; ${ }^{6}$ Institute of Statistics, National Chung Hsing University, Taichung, Taiwan

Correspondence: Chih-Ming Lin, Department of Neurology, Changhua Christian Hospital, 135 Nanhsiao Street, Changhua City, 50006, Taiwan, Email josephsimion@gmail.com; 166110@cch.org.tw; Ying-Lin Hsu, Department of Applied Mathematics, National Chung Hsing University, Taichung, Taiwan Email ylhsu@nchu.edu.tw

Background: Middle cerebral artery (MCA) ischemic stroke poses a major threat to human beings and prompts intravenous thrombolytic and/or thrombectomy management remains the gold standard treatment. However, not all MCA stroke patients fit in the inclusion and exclusion criteria that many patients only receive conventional medical therapy. We attempt to seek the baseline parameters that can effectively predict patients' long-term functionality, as well as hypothesizing that the carotid duplex derived resistance/pulsatility index might be capable of fulfilling this purpose.

Methods: The $741 \mathrm{MCA}$ ischemic stroke patients have been retrospectively recruited for the project. Under the initial screening, matching the inclusion and exclusion criteria, there are 471 participants to be enrolled in the study. The patients' basic information, along with outcome assessments, pre-admission Barthel index and NIHSS score, and pre- and post-treatment mRS are recorded. All statistical analyses were performed using $\mathrm{R}$ for Windows (version 3.6.3). The significance level was set at $\mathrm{P}<0.05$ for all analyses. Results: Of the 471 patients, 239 participants show a net mRS improvement, whereas the other 232 show deterioration. Hyperlipidaemia, chronic kidney disease, and dementia are related to long-term functionality improvement. The multivariate logistic regression analysis shows that right common carotid artery (CCA) resistance index (RI) and ischemic heart disease play a significant role in favourable outcome functionality. The ROC and Youden Index models are formulated, and it shows that Barthel Index and the NIHSS are most significant in the outcome measurement group (AUC: 0.675, 0.653; cut-off point: 57.5, 3.5, respectively). The rightside CCA RI is the solely important outcome predictor for the baseline carotid duplex study (AUC: 0.5 ; cut-off point: 0.785 ).

Conclusion: The favourable long-term functionality of MCA ischemic stroke patients receiving conventional medical treatment seems to correlate fairly with pre-admission NIHSS and Barthel index scores. Underlying hyperlipidaemia, chronic kidney disease, and dementia are conversely associated with favourable long-term capability. Moreover, the value of CCA RI appears to significantly alter the long-term outcomes in this group of patients.

Keywords: middle cerebral artery, ischemic stroke, thrombolytic therapy, thrombectomy, Barthel Index, NIHSS, mRS, common carotid artery, resistance index

\section{Introduction}

Ischemic stroke represents the third major cause of morbidity and mortality worldwide and frequently occurs in Taiwan. ${ }^{1}$ The aetiology of ischemic stroke is heterogeneous and can be hard to deal with if the source of the malady is not found completely. There are several subtypes of stroke, apart from brain stem stroke, the disease progression of middle cerebral artery (MCA) infarction being the most unpredictable and thus requiring aggressive management. ${ }^{1,2}$ In the first week after an index event, the cerebral infarct core, as well as the penumbra regions, might cause cellular oedematous change 
leading to expansion of the brain parenchyma, and to a cerebral herniation. ${ }^{3}$ Based on the American Heart Association/ American Stroke Association guidelines, careful observation and prompt treatment of intravenous thrombolytic/ thrombectomy therapies are the gold standard protocols if stroke sufferers of whom fitted into the inclusion and exclusion criteria. ${ }^{4}$ Nevertheless, there are still some problems that remained to be solved. Firstly, first time MCA stroke episodes stand as more emergent situations and clinical symptoms and presentation signs are vaguer, mainly because an in-charge clinician is unlikely to understand the cause of the stroke episode, as compared to a recurrent stroke episode, by which the underlying mechanism is much clearer. This clinical scenario can potentially lead to missing out on the "gold therapeutic time window" meaning that the time is no longer suitable for invasive management. ${ }^{5,6}$ Secondly, even though patients may arrive within the "gold therapeutic time window", through meticulous evaluation by the neurologist, based on the NIHSS score and the intravenous thrombolytic/ thrombectomy inclusion and exclusion criteria, some patients do not fit into the evaluation panel and cannot receive appropriate treatment. Reviewing the thrombolytic guideline for inclusion and exclusion criteria, the proper NIHSS scores for potential candidates are between 4 and 25 points. In addition, those with poor body function and with previous anticoagulant medication intake cannot have this treatment administered and are thus excluded from thrombolytic treatment (r-tPA) ${ }^{7-9}$ This group of MCA patients are subsequently left with traditional conservative medical treatment of antiplatelet therapy and attendance in the intensive care unit in the first week to prevent fluctuating unstable vital signs. This can therefore be considered a clinical dilemma, as first line neurologists cannot predict patient long-term functionality, meaning it is very difficult for them to explain to a patient's family members, either in detail or to any certainty, the patient's neurological sequelae. The carotid duplex exam is a noninvasive, low-cost, and repeatable examination that could be suitable for ischemic stroke patients no matter their physical condition. This portable machine can be carried to and operated in the proximity of a patient's bed revealing their current condition in real-time. However, despite the above-mentioned benefits and the other obvious and evident advantages of the carotid duplex, clinical stroke neurologists often neglect the importance of this clinical neuroimaging modality. ${ }^{10,11}$ In this current investigation our team recruited a series of middle cerebral artery ischemic stroke patients receiving conventional medical treatment (not intravenous thrombolytic/thrombectomy) at the triage section of the emergency department. This group of patients went through standard stroke protocol therapy. Their baseline demographics, biochemistry, neuroimaging studies, and carotid duplex exam reports are well documented. The primary goal of the current investigation is to define and to see whether any powerful surrogates exist in the baseline information that can project and effectively predict a patient's long-term outcome (evaluated by the mRS, pre and post admission of treatment from the department of neurology). Secondly, we attempted to look for any underlying comorbidities that could potentially devastate the index stroke episode, which will remind neurologists to aggressively treat the disease at the same time in order to eliminate poor consequences. Thirdly, as the carotid duplex exam is a routine clinical neuroimaging modality in our hospital, carried out within 3 days of admission, using carotid ultrasound datasets, we wish to see if carotid duplex parameters (the resistance index and the pulsatility index) can play a major role in determining early outcomes for a patient, as well as in establishing important cut-off point values.

\section{Materials and Methods}

\section{Candidate Selection}

In this investigation, the authors retrospectively studied the data from 741 middle cerebral artery ischemic stroke patients, with the help of the Department of Information Management at Changhua Christian Hospital (CCH), collecting patient datasets and medical records for the 13 periods, January 2010 to January 2020. Although the patient list was completed and provided by the Department of Information and Management, the outcome assessments, neuroimaging studies and carotid duplex datasets were manually collected and recorded in full Excel files. The complete datasets are stored in the study room on the fourth floor of the department of physical medicine and rehabilitation in the $\mathrm{CCH}$. The patients were screened, and ultimately, there were 471 participants, based on the predefined inclusion and exclusion criteria. All of the subjects were enrolled through the triage section of the emergency department (ED) and were evaluated by a stroke neurologist and ED doctor, and were not considered thrombolytic and/or thrombectomy treatment candidates. The participants were subsequently admitted to the neurological intensive care unit for close monitoring before being 
transferred to a general ward for advanced treatment and then discharged for outpatient follow-ups for a period of one year (12 months) or more. The clinical information collected was cross-checked to ensure consistency between the original (paper) medical records and the electronic information stored on the Changhua Christian Hospital 2000 computer-based medical record systems network. The study protocol and methodology are described in the flow chart (Figure 1). The study protocol was approved by the Research Ethics Committee, Changhua Christian Hospital (certificate: 201121). Informed consent was waived as patient's personal information was detached from the raw datasets.

All patients included in the study met the following pre-defined inclusion and exclusion criteria:

a) Participants were between 20 and 90 years of age, with evidence of first time middle cerebral artery ischemic stroke at the index event and evaluated with cerebral computed tomography (CT), angiography (CTA), CT perfusion (P), and/or cerebral magnetic resonance imaging not suitable for intravenous thrombolytic therapy and/or thrombectomy treatment.

b) There was no observation of recurrent cerebral or other vascular events during the study period.

c) Participants had no severe carotid stenosis/vertebral stenosis or had ever received carotid endarterectomy or carotid stenting, excluding other subtypes/aetiologies of stroke mechanism and also excluding the possibility of cardiogenic emboli.

d) Participants had completed at least 12 months of follow-up after treatment.

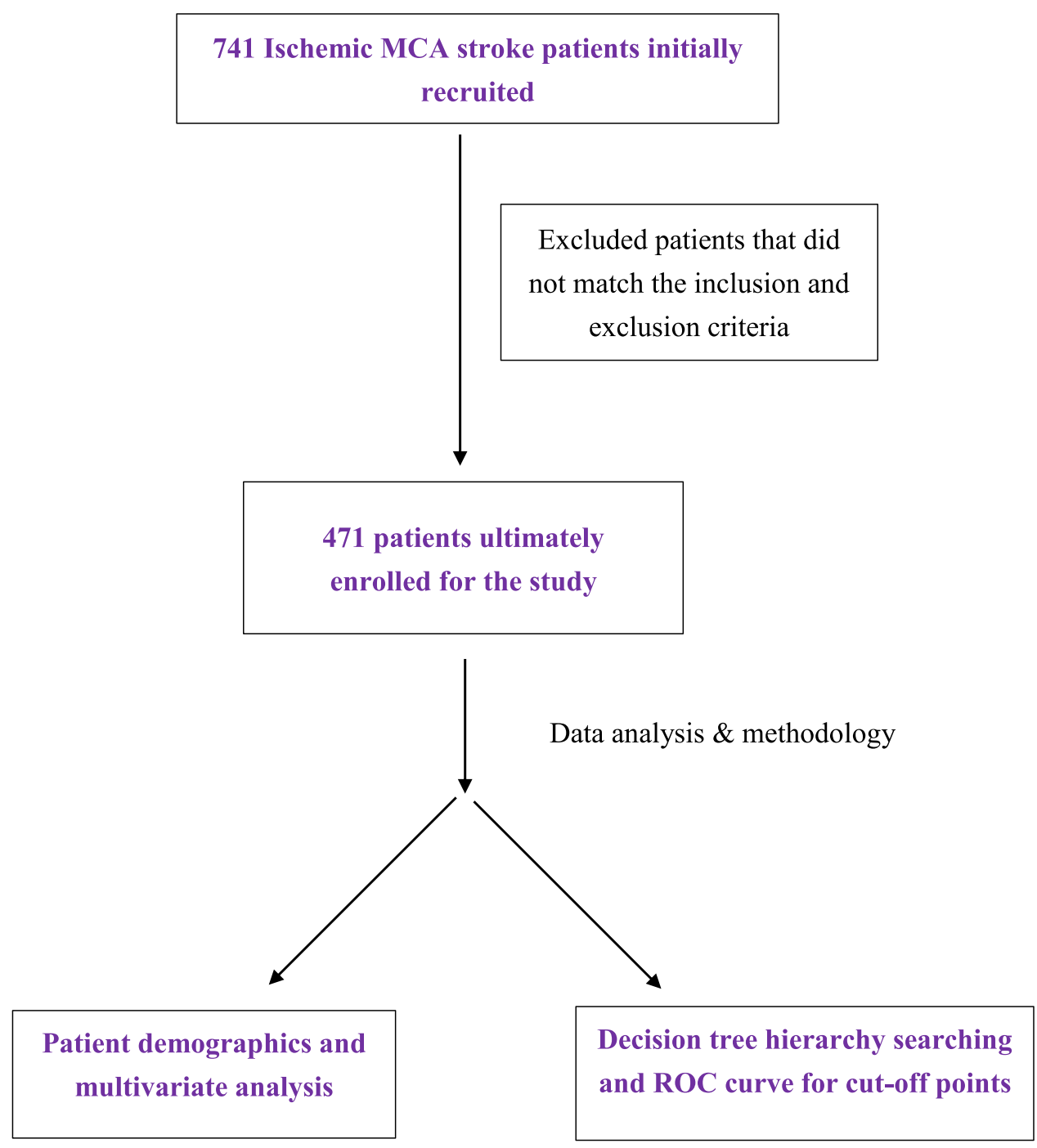

Figure I Study flow chart. 
e) Participants were admitted through conventional antiplatelet therapy, with close monitoring of their vital signs from the intensive care unit through a general ward before subsequently being discharged from a neurological ward.

The exclusion criteria were:

a) NIHSS score $<4$ or $>25$ points during initial evaluation, and/or recurrent stroke of the index stroke episode.

b) Participants receiving long-term anticoagulant therapy before the index event and/or already known to possibly have internal medical diseases.

c) Participants with cerebral bleeding based on the cerebral tomography scan.

d) Participants with cerebral arterio-venous malformations or aneurysms.

e) Participants with a recurrent stroke during the study period.

f) Participants demonstrating systemic vascular events or recurrent cerebral vascular episodes.

Baseline biochemistry and neuro-radiological exams were carried out in both the emergency room and in the neurological ward. The comorbidities, neurological and physical exams, relevant drug history, and patient personal history were carefully recorded during the hospitalization period. The carotid duplex exam was conducted within 3 days of initial admission to hospital. All of the parameters of the carotid ultrasound were documented. The patient's stroke severity/physical condition was recorded once using the NIHSS score and the Barthel Index upon admission to the ED, with evaluation by both in charge neurologist and ED doctor. The purpose of it is to make sure the genuine scenario whether the patients' suitability for intravenous thrombolytic therapy and/or thrombectomy is valid.

In addition to the NIHSS score and the Barthel Index, the CCH protocol utilizes the modified Rankin Scale (mRS) to follow-up patients' general condition in the long run. We follow up patients' mRS status upon admission to the ED, 3, 6, and up to 12-month time in the outpatient clinic settings after neurological ward discharge. Each assessment was conducted by the same neurologist. In the meantime, the ED doctor was also involved in this measurement to ensure the accuracy. If they contained any discrepancy in-between, a third party (usually the stroke program case manager) would be invited to validate the recordings. The modified Rankin Scale (mRS) is a commonly used scale for measuring the degree of disability or dependence in the daily activities of people who have suffered a neurological disability from a stroke or from other causes. The scale runs from 0 (perfect health without symptoms) through to 6 (death). The information collected may improve our ability to prognosticate the outcome. Stroke patients were stratified based on their net mRS score.

\section{Cervical Carotid Duplex Exam}

All procedures were conducted by a stroke neurologist team in a specialized angiography clinic at the Changhua Christian Hospital, Changhua, Taiwan. Cervical carotid artery ultrasound examination (Philips iE33 7-Mhz linear transducer) was performed upon a patient upon arrival at the emergency room. Cross-sectional B-mode scanning was performed to check for intraluminal plaque material and the longitudinal screening method was adopted to confirm the presence of plaque. Two physicians assessed and classified plaques into subtypes 1, 2, 3, or 4, according to the International Classification System. ${ }^{12}$ Whenever physicians disagreed, a third physician would make the final assessment. The intima-media thickness of the mid-portion of the CCA was measured on the ipsilateral side of the index stroke event. The parameters of the peak systolic velocity (PSV), end-diastolic velocity (EDV), resistance index (RI) (calculated as $[\mathrm{PSV}-\mathrm{EDV}] / \mathrm{PSV}$ ) and pulsatility index (PI) (calculated as [PSV-EDV]/mean of the velocity) of the CCA, ICA, ECA, VA, and OA were measured bilaterally. The reversal of blood flow in the OA was also measured. Forward flow was defined as blood flow detected out of the stenotic ipsilateral carotid artery, whereas reverse flow was defined as blood flow into the carotid artery. ${ }^{13-15}$ The machine (Philips iE33 7) automatically calculated the plaque index on both the ipsilateral and contralateral sides of the cerebral lesion.

\section{Cerebral Computed Tomography (CT)/Angiography (CTA) and/CT Perfusion (P)}

Multiple sequential axial images were obtained from the base of the skull up through the vertex without intravenous contrast material. The technical parameters were as follows: thickness, $5 \mathrm{~mm}$; length, $200 \mathrm{~mm}$; increment, $10 \mathrm{~mm}$; $\mathrm{kV}$, 120; mA, 550. CTA examinations were performed using a second-generation dual-source CT scanner (SOMATOM Definition Flash, Siemens Healthcare, Forchheim, Germany). After placing a 18-gauge intravenous catheter in the 
antecubital vein, $100 \mathrm{~mL}$ of Iodixanol (Visipaque 320, GE Healthcare, Carrigtwohill, Ireland) or Iohexol (Omnipaque 350, GE Healthcare, Ireland) was infused as a contrast medium at a rate of $5 \mathrm{~mL} / \mathrm{s}$. The initial injection delay was estimated using the bolus-tracking technique, where the threshold was 100 Hounsfield units. Scanning was performed using a dual-energy mode with pitch, rotation time, and collimations of $0.9,0.28 \mathrm{~s}$, and $2 \mathrm{~mm} \times 32 \mathrm{~mm} \times 0.6 \mathrm{~mm}$, respectively, at $100 \mathrm{kV} / 150$ ref. mAs (Tube A) and Sn140 kV/178 ref. mAs (Tube B). The area from the aortic arch to the top of the neurocranium was scanned. CT images were reconstructed at a slice thickness of $0.6 \mathrm{~mm}$ and at an increment of $0.3 \mathrm{~mm}$ with a medium-smooth kernel. CTP scans were subsequently performed with a contrast bolus of $50 \mathrm{~mL}$ of Omnipaque 350 (GE Healthcare, Milwaukee, WI, USA). Perfusion data sets were postprocessed using a Siemens Multimodality Workplace Workstation (Siemens Medical, Germany), which calculated mean transit time (MTT), cerebral blood volume (CBV), cerebral blood flow (CBF), and time to peak (TTP). The arterial input and venous outflow curves were analyzed to ensure data set completeness. The CTP parameters are defined as follows:

1. dMTT: ipsilateral MTT-contralateral MTT

2. MTT ratio: ipsilateral MTT/contralateral MTT

3. MTT index: (ipsilateral MTT-contralateral MTT)/contralateral MTT

4. dCBV: ipsilateral $\mathrm{CBV}-$ contralateral $\mathrm{CBV}$

5. $\mathrm{CBV}$ ratio: ipsilateral $\mathrm{CBV} /$ contralateral $\mathrm{CBV}$

6.The CBV index: (ipsilateral $\mathrm{CBV}$-contralateral $\mathrm{CBV}$ )/contralateral $\mathrm{CBV}$

7. $\mathrm{dCBF}$ : ipsilateral $\mathrm{CBF}-$ contralateral $\mathrm{CBF}$

8. $\mathrm{CBF}$ ratio: ipsilateral $\mathrm{CBF} /$ contralateral $\mathrm{CBF}$

9. $\mathrm{CBF}$ index: (ipsilateral $\mathrm{CBF}-$ contralateral $\mathrm{CBF}$ )/contralateral $\mathrm{CBF}$

10. dTTP: ipsilateral TTP-contralateral TTP

11. TTP ratio: ipsilateral TTP/contralateral TTP

12. TTP index: (ipsilateral TTP-contralateral TTP)/contralateral TTP

\section{Magnetic Resonance Imaging and Angiography (MRI/A)}

Structural and functional MR imaging and angiographic examinations were performed using a 3-T (Magnetom Verio, Siemens Healthcare, USA) or a 1.5-T imager (Magnetom Aera, Siemens Healthcare) with a cervical coil. A standard protocol for evaluating a stroke, including axial DWI, apparent diffusion coefficient, and fluid-attenuated inversion-recovery sequences was followed. Three-dimensional TOF MR angiography without contrast enhancement was performed in the transverse plane using a sliding interleaved $\mathrm{kY}$ acquisition sequence comprising 6 overlapping slabs of 11 sections, with the following parameters: section thickness, $1.2 \mathrm{~mm}$; repetition time (milliseconds)/echo time (milliseconds), 242/7; flip angle, $20^{\circ}$; field of view, $200 \times 200 \mathrm{~mm}$; matrix, $205 \times 320$. The final pixel size was $0.975 \mathrm{~mm} \times 0.625 \mathrm{~mm}$. Contrast-enhanced MR angiography was not routinely performed.

\section{Statistical Analysis}

All statistical analyses were performed using $\mathrm{R}$ for Windows (version 3.6.3). The significance level was set at $\mathrm{P}<0.05$ for all analyses. Before and after hospitalization, mRS scores were compared to determine patient outcomes and classified as improved and unimproved.

To test the association between the degree of disability and variables before hospitalization, with the degree of disability (Net mRS) defined as (after hospitalization mRS Scores) - (before hospitalization mRS scores). Negative (mRS $[-])$ scores $(<0)$ indicated improvement and positive or stationary $(\mathrm{mRS}[+])$ scores $(\geq 0)$ indicated no improvement in patient condition. A Mann-Whitney $U$-test was used to determine differences in the mean values of continuous variables between the two groups. Categorical variables were compared using the chi-squared test of goodness of fit. The chisquare independence test was used to explore the relationship between Net mRS and disease and Yule'sQ ${ }^{16}$ was used to calculate the correlation coefficient. Multivariate logistic regression analysis was modelled through stepwise regression and employed to assess the significance of risk factors and to obtain odds ratios (ORs). 
The decision tree ${ }^{17}$ was formulated to weigh the importance of each variable. This can reveal the most critical risk factors in both groups, thereby assisting clinicians in deciding patient outcomes. The methodology uses a pre-pruning strategy; a node is split only if a significant criterion is satisfied, thus preventing overfitting. The node cut out from the first level of the decision tree was regarded as the cut point. Data lower than the cut point were set to 1, with data greater than or equal to the cut point being set to 0 .

Using the receiver operating characteristic (ROC) curve to find the cut-off point, the area under the curve (AUC) indicates the overall accuracy of the degree of disability and the variables before hospitalization. Youden's index was equal to sensitivity + (1-specificity), and the largest Youden's index was chosen to determine the cut-off point. Sensitivity, specificity, positive predictive value (PPV), negative predictive value (NPV), positive likelihood ratio (+LR) and negative likelihood ratio (-LR) were calculated using true positive (TP), true negative (TN), false negative (FN) and false positive (FP), respectively, representing the true positive rate, true negative rate, true positive detection rate, true negative detection rate, the ratio of true-positive rate to false-positive rate, and the ratio of true-negative rate to false-negative rate. The model is set to find the predicted value and uses the predicted value to run the ROC curve to seek for the most suitable segmentation probability. A predicted value less than this probability is classified as 0 , and one greater than it is classified as 1 , to calculate the confusion matrix in a mathematical manner. The decision tree utilizes the Gini index to classify variables. Its purpose is to find the cut point to divide the variables into two categories (Improvement or non-improvement group).

\section{Results}

Of the 471 patients, Table 1 shows their basic demographics and any previous underlying disease. Two hundred and thirty-nine patients show net mRS improvement, whereas 232 patients show deterioration. Regarding personal information, there are: 273 men (58.0\%), 198 women (42.0\%); with 313 having an education level below that of middle school (66.5\%); with 343 being unmarried (72.8\%); and, for the disease part, there were 328 patients $(69.6 \%)$ with hyperlipidemia, 224 patients (47.6\%) with heart disease, 194 patients (41.2\%) with diabetes, 165 patients $(35.0 \%)$ with malignant

Table I Baseline Patients' Demographics for mRS Improvement and Non-Improvement Groups

\begin{tabular}{|c|c|c|c|c|c|c|c|c|}
\hline \multicolumn{9}{|c|}{ Descriptive Statistics and Test of Discrete Variables } \\
\hline & & & \multirow[t]{2}{*}{ Overall N (\%) } & \multicolumn{2}{|c|}{$\begin{array}{l}\text { Non-Improvement } \\
\text { mRS }(n=232)\end{array}$} & \multicolumn{2}{|c|}{$\begin{array}{l}\text { Improvement mRS } \\
\qquad(n=239)\end{array}$} & \multirow[t]{2}{*}{ p-value } \\
\hline & & & & $\mathbf{N}$ & $\%$ & $\mathbf{N}$ & $\%$ & \\
\hline \multirow[t]{6}{*}{ Personal Information } & \multirow[t]{2}{*}{ Gender } & Male & $273(58.0)$ & 133 & 57.3 & 140 & 58.60 & 0.672 \\
\hline & & Female & $198(42.0)$ & 99 & 42.7 & 99 & 41.42 & 1.000 \\
\hline & \multirow[t]{2}{*}{ Education } & Elementary School & $313(66.5)$ & 160 & 82.5 & 153 & 81.40 & 0.692 \\
\hline & & Junior High School & $69(14.6)$ & 34 & 17.5 & 35 & 18.62 & 0.904 \\
\hline & \multirow[t]{2}{*}{ Marital status } & Married & $128(27.2)$ & 70 & 30.2 & 58 & 24.30 & 0.289 \\
\hline & & Unmarried & $343(72.8)$ & 162 & 69.8 & $|8|$ & 75.73 & 0.305 \\
\hline \multirow[t]{8}{*}{ Underlying diseases } & \multicolumn{2}{|c|}{ Essential hypertension } & $60(12.7)$ & 34 & 14.7 & 26 & 10.9 & 0.302 \\
\hline & \multicolumn{2}{|c|}{ Mixed type hyperlipidaemia } & $328(69.6)$ & 175 & 75.4 & 153 & 64.0 & 0.225 \\
\hline & \multicolumn{2}{|c|}{ Diabetes Mellitus } & $194(4 \mid .2)$ & 105 & 45.3 & 89 & 37.2 & 0.251 \\
\hline & \multicolumn{2}{|c|}{ Chronic heart failure } & $224(47.6)$ & 109 & 47 & 115 & 48.1 & 0.689 \\
\hline & \multicolumn{2}{|c|}{ Chronic Kidney Disease } & $127(27.0)$ & 73 & 31.5 & 54 & 22.6 & 0.092 \\
\hline & \multicolumn{2}{|c|}{ Dementia } & $57(12.1)$ & 36 & 15.5 & 21 & 8.8 & $0.047 *$ \\
\hline & \multicolumn{2}{|c|}{ Depression } & $48(10.1)$ & 25 & 10.8 & 23 & 10 & 0.773 \\
\hline & \multicolumn{2}{|c|}{ Insomnia/Anxiety } & $93(19.7)$ & 45 & 19.4 & 48 & 20 & 0.756 \\
\hline
\end{tabular}

Notes: After hospitalization mRS - Before hospitalization $\mathrm{mRS}, \geq 0$, indicating non-improvement or stationary and $<0$ indicating improvement in patient condition; *p-value $<0.05$ significant, Test of Goodness-of-Fit.

Abbreviations: mRS, modified Rankin Scale; N, number. 
tumors, and $127(27.0 \%)$ patients with kidney disease. The underlying disease of dementia shows a statistical significance $(\mathrm{P}<0.05)$.

Table 2 shows other basic patient information before admission for receipt of stroke therapy. Hospital days ( $\mathrm{p}$-value $=$ $0.008<0.05$ ), showing whether there was a significant difference in the hospital days for mRS improvement or not. The median hospital stay with an improved disability was 10 days, while the median hospital stay with no improvement in disability was 8 days. For NIHSS (p-value $<0.05$ ), there was a significant difference in NIHSS with or without mRS improvement. The median NIHSS with an improved disability was 7 points, and the median with no improvement in disability was 3 points. For the Barthel Index ( $p$-value $<0.05$ ), there is a significant difference in the Barthel Index depending on whether there is an mRS improvement or not. The median of the Barthel Index with improved disability was 40 points, and the median of the Barthel Index with no improvement in disability was 70 points. For the number of comorbidities $(p$-value $=0.007<0.05)$, there was a significant difference in the number of comorbidities between the two groups if the mRS was improved. There were 2 types of comorbidities with improved disability, and the median number of types for comorbidities without improved disability was 3 . HbA1C (p-value $=0.048<0.05$ ), no matter whether mRS improves or not, there is a significant difference in HbA1C between the two groups. The median HbA1C with improved disability was 5.8, and the median $\mathrm{HbA1C}$ with no improvement was 5.9. For the total cholesterol level ( $\mathrm{p}$-value $=0.017<0.05$ ), there is a significant difference in total cholesterol between the two groups if $\mathrm{mRS}$ is improved. The median value with improved disability was 154, and the group with no improvement was 163 . For white blood cell count $(\mathrm{WBC})(\mathrm{p}$-value $=0.008<0.05)$, no matter whether $\mathrm{mRS}$ improves or not, there is a significant difference in WBC between the two groups. The median WBC with improved disability was 7.9, and the median WBC with no improvement was 7.1 .

In Table 3, we attempt to find the association by: weighing various comorbidities, correlating between degree of disability, underlying diseases and the net mRS.

The table reveals that hyperlipidaemia $(p$-value $=0.0095<0.05)$, chronic kidney disease $(p$-value $=0.0389<0.05)$, and dementia ( $\mathrm{p}$-value $=0.0359<0.05$ ) are associated with long-term functionality improvement. With weighing of the above mentioned, all three are inversely correlated, with dementia being the most powerful $(0.16)$, followed by mixedtype hyperlipidaemia (0.136), and chronic kidney disease $(0.113)$.

The multivariate logistic regression data analysis is displayed in Table 4. The result is achieved via stepwise regression to select variables, under control of other variables; it shows right common carotid artery resistance index ( $\mathrm{p}$-value $=0.04<0.05)$, for every additional unit, and the odds ratio for improvement of the degree of disability is $3.56 * 10^{-7}$ times the original. In addition, coronary heart disease $((\mathrm{p}$-value $=0.0016<0.05))$ also demonstrates statistical significance. The odds ratio for coronary artery disease is 0.357 times that of an improvement over non-improvement groups.

Table 5 shows the decision tree data analysis. Using the carotid duplex variables, left CCA RI and PI, and right CCA RI and PI, ECA RI show statistical significance. Using basic patient biochemistry, it shows that total cholesterol, HbA1C, LDL, WBC, CPK, K, and GOT are important variables. As for personal demographics, it elucidates that: the Barthel Index, the number of comorbidities, hospital days, and NIHSS are crucial predictors.

Among the most important predictors, we use the ROC and Youden Index model to find the predicted value, correlating with the next $\mathrm{mRS}$ to find the most suitable segmentation probability. For calculation of the confusion matrix, a predicted value less than this probability is classified as 0 , while one greater than it is classified as 1 . The findings are shown on Table 6 and Appendix 1. Among the outcome measurements, the Barthel Index and NIHSS are the most significant (AUC: 0.675, 0.653; cut-off point: 57.5, 3.5, respectively). Among the blood chemicals, HbA1C, total cholesterol and WBC are crucial variables (AUC: 0.561, 0.573, 0.578; cut-off point: 6.15, 164.5, 7.25 respectively). As for personal information, hospital days and number of comorbidities are crucial items (AUC: 0.571, 0.569; cut-off point: $6.5,2.5$, respectively). The right-side CCA RI is the sole important outcome predictor for the baseline carotid duplex study (AUC: 0.5; cut-off point: 0.785). 
Table 2 Comparison of Baseline Biochemistry Outcome Measurements and Neuroimaging Studies for the mRS Two Groups

\begin{tabular}{|c|c|c|c|c|c|c|c|c|}
\hline \multicolumn{9}{|c|}{ Descriptive Statistics and Test of Continuous Variables } \\
\hline & \multirow[t]{2}{*}{ Overall Median (Range) } & \multicolumn{3}{|c|}{ Non-Improvement mRS $(n=232)$} & \multicolumn{3}{|c|}{ Improvement mRS (n=239) } & \multirow[t]{2}{*}{ p-value } \\
\hline & & Min & Median & Max & Min & Median & Max & \\
\hline \multicolumn{9}{|l|}{ Personal Information } \\
\hline Age & $69(22-90)$ & 22 & 69 & 90 & 28 & 69 & 90 & 0.585 \\
\hline Hospital days & $9(2-121)$ & 2 & 8 & 121 & 2 & 10 & 92 & $0.008^{*}$ \\
\hline \multicolumn{9}{|l|}{ Outcome Measurement } \\
\hline Admission NIHSS & $5(0-38)$ & 0 & 3 & 38 & 0 & 7 & 37 & $<0.000 I^{*}$ \\
\hline Admission Barthel Index & $50(0-100)$ & 0 & 70 & 100 & 0 & 40 & 100 & $<0.000 I^{*}$ \\
\hline \multicolumn{9}{|l|}{ Disease } \\
\hline Number of comorbidities & $3(0-10)$ & 0 & 3 & 10 & 0 & 2 & 9 & $0.007^{*}$ \\
\hline \multicolumn{9}{|l|}{ Blood Chemicals } \\
\hline LDL & $10 \mid(20-284)$ & 36 & 106.5 & 284 & 20 & 98 & 212 & 0.111 \\
\hline GOT & $26(9-748)$ & 9 & 27 & 748 & 13 & 26 & 99 & 0.605 \\
\hline $\mathrm{HbAIC}$ & $5.8(4-13.7)$ & 4 & 5.9 & 12.8 & 4.3 & 5.8 & 13.7 & $0.048^{*}$ \\
\hline Blood Sugar before meal & $128(46-666)$ & 46 & $|3|$ & 666 & 46 & 128 & 413 & 1.000 \\
\hline Total cholesterol & $158(55-426)$ & 55 & 163 & 426 & 63 & 154 & 289 & $0.017^{*}$ \\
\hline CPK & 71 (16-1938) & 16 & 69 & 1938 & 23 & 74 & 1006 & 0.128 \\
\hline WBC & $7.5(1.8-20.5)$ & 1.8 & 7.1 & 20.5 & 3.2 & 7.9 & 15.6 & $0.008^{*}$ \\
\hline K & $3.8(2.7-5.6)$ & 2.7 & 3.8 & 5.6 & 2.8 & 3.8 & 5.5 & 0.604 \\
\hline \multicolumn{9}{|c|}{ CDS carotid duplex examination } \\
\hline \multicolumn{9}{|l|}{ Right } \\
\hline CCA.PI & $1.73(0.8 \mathrm{I}-3.94)$ & 0.86 & 1.72 & 3.67 & 0.81 & 1.73 & 3.94 & 0.937 \\
\hline ECA.PI & $2.75(1.34-7.38)$ & 1.34 & 2.79 & 7.38 & 1.52 & 2.74 & 7.07 & 0.767 \\
\hline CCA.RI & $0.76(0.55-1)$ & 0.55 & 0.76 & 1 & 0.55 & 0.76 & 0.99 & 0.976 \\
\hline ECA.RI & $0.88(0.7-1)$ & 0.7 & 0.87 & 1 & 0.71 & 0.88 & 1 & 0.955 \\
\hline \multicolumn{9}{|l|}{ Left } \\
\hline CCA.PI & $1.765(0.92-5.66)$ & 1.07 & 1.78 & 5.66 & 0.92 & 1.75 & 3.25 & 0.492 \\
\hline ECA.PI & $2.59(1.22-10.7 \mid)$ & 1.22 & 2.58 & $10.7 \mid$ & 1.34 & 2.59 & 6.58 & 0.586 \\
\hline CCA.RI & $0.77(0.58-1)$ & 0.63 & 0.77 & 1 & 0.58 & 0.765 & I & $0.56 I$ \\
\hline ECA.RI & $0.87(0.65-1)$ & 0.65 & 0.87 & I & 0.7 & 0.87 & I & 0.696 \\
\hline
\end{tabular}

Notes: After hospitalization mRS - Before hospitalization mRS, $\geq 0$, indicating non-improvement or stationary and $<0$ indicating improvement in patient condition; *p-value<0.05 significant, Mann-Whitney's U-test.

Abbreviations: mRS, Modified Rankin Scale; NIHSS, National Institute of Health Stroke Scale; N, number; LDL, low-density lipoprotein; GOT, Glutamic Oxaloacetic Transaminase; HbAIC, hemoglobin Al c; Sugar, sugar before meals; T_CHO, Total Cholesterol; CPK, creatine phospho kinase; WBC, White Blood Cell; CDS, carotid duplex scan; CCA, common carotid artery; ECA, external carotid artery; $\overline{\mathrm{PI}}$, perfusion Index; RI, resistance index. 
Table 3 Correlation Between mRS Improvement and Underlying Diseases

\begin{tabular}{|c|c|c|c|c|c|c|c|c|c|}
\hline \multicolumn{10}{|c|}{ Correlation Between Degree of Disability and Underlying Diseases } \\
\hline \multicolumn{2}{|l|}{ Diseases } & \multicolumn{2}{|c|}{ Overall } & \multicolumn{2}{|c|}{ Improvement mRS } & \multicolumn{2}{|c|}{ Non-Improvement mRS } & \multirow[t]{2}{*}{ p-value } & \multirow[t]{2}{*}{ Yule's Q } \\
\hline & & $\mathbf{N}$ & $\%$ & $\mathbf{N}$ & $\%$ & $\mathbf{N}$ & $\%$ & & \\
\hline Essential hypertension & $\begin{array}{l}+ \\
-\end{array}$ & $\begin{array}{c}60 \\
411\end{array}$ & $\begin{array}{l}12.739 \\
87.261\end{array}$ & $\begin{array}{c}26 \\
213\end{array}$ & $\begin{array}{l}10.879 \\
89.121\end{array}$ & $\begin{array}{c}34 \\
198\end{array}$ & $\begin{array}{l}14.655 \\
85.345\end{array}$ & 0.2754 & -0.085 \\
\hline Mixed type hyperlipidaemia & $\begin{array}{l}+ \\
-\end{array}$ & $\begin{array}{l}328 \\
143\end{array}$ & $\begin{array}{l}69.639 \\
30.361\end{array}$ & $\begin{array}{l}153 \\
86\end{array}$ & $\begin{array}{l}64.017 \\
35.983\end{array}$ & $\begin{array}{c}175 \\
57\end{array}$ & $\begin{array}{l}75.431 \\
24.569\end{array}$ & $0.0095^{*}$ & -0.136 \\
\hline Diabetes mellitus & $\begin{array}{l}+ \\
-\end{array}$ & $\begin{array}{l}194 \\
277\end{array}$ & $\begin{array}{l}41.189 \\
58.811\end{array}$ & $\begin{array}{c}89 \\
150\end{array}$ & $\begin{array}{l}37.238 \\
62.762\end{array}$ & $\begin{array}{l}105 \\
127\end{array}$ & $\begin{array}{l}45.259 \\
54.741\end{array}$ & 0.094 & -0.083 \\
\hline Chronic heart failure & + & $\begin{array}{l}224 \\
247\end{array}$ & $\begin{array}{l}47.558 \\
52.442\end{array}$ & $\begin{array}{l}115 \\
124\end{array}$ & $\begin{array}{l}48.117 \\
51.883\end{array}$ & $\begin{array}{l}109 \\
123\end{array}$ & $\begin{array}{l}46.983 \\
53.017\end{array}$ & 0.8775 & 0.011 \\
\hline Chronic Kidney disease & + & $\begin{array}{l}127 \\
344\end{array}$ & $\begin{array}{l}26.964 \\
73.036\end{array}$ & $\begin{array}{c}54 \\
185\end{array}$ & $\begin{array}{l}22.594 \\
77.406\end{array}$ & $\begin{array}{c}73 \\
159\end{array}$ & $\begin{array}{l}31.466 \\
68.534\end{array}$ & $0.0389 *$ & -0.113 \\
\hline Dementia & + & $\begin{array}{c}57 \\
414\end{array}$ & $\begin{array}{l}12.102 \\
87.898\end{array}$ & $\begin{array}{c}21 \\
218\end{array}$ & $\begin{array}{c}8.787 \\
91.213\end{array}$ & $\begin{array}{c}36 \\
196\end{array}$ & $\begin{array}{l}15.517 \\
84.483\end{array}$ & $0.0359 *$ & -0.16 \\
\hline Depression & $\begin{array}{l}+ \\
-\end{array}$ & $\begin{array}{c}48 \\
423\end{array}$ & $\begin{array}{l}|0.19| \\
89.809\end{array}$ & $\begin{array}{c}23 \\
216\end{array}$ & $\begin{array}{c}9.623 \\
90.377\end{array}$ & $\begin{array}{c}25 \\
207\end{array}$ & $\begin{array}{l}10.776 \\
89.224\end{array}$ & $0.794 I$ & -0.031 \\
\hline Insomnia & $\begin{array}{l}+ \\
-\end{array}$ & $\begin{array}{c}93 \\
378\end{array}$ & $\begin{array}{l}19.745 \\
80.255\end{array}$ & $\begin{array}{l}48 \\
191\end{array}$ & $\begin{array}{l}20.084 \\
79.916\end{array}$ & $\begin{array}{c}45 \\
187\end{array}$ & $\begin{array}{l}19.397 \\
80.603\end{array}$ & 0.943 & 0.011 \\
\hline
\end{tabular}

Notes: Chi-Square Test of Independence; After hospitalization mRS - Before hospitalization $\mathrm{mRS}$, $\geq 0$, indicating non-improvement or stationary and $<0$ indicating improvement in patient condition; *p-value $<0.05$ significant; yule's $\mathrm{Q}$, Correlation coefficient, $-\mathrm{I} \leq \mathrm{Q} \leq \mathrm{I}$.

Abbreviations: mRS, modified Rankin Scale; +, yes; -, no; N, number.

Table 4 Multivariate Logistic Analysis Selecting Predictors

\begin{tabular}{|c|c|c|c|c|c|}
\hline \multicolumn{6}{|c|}{ Multivariate Logistic Regression } \\
\hline Dependent Variable & Predict Variable & OR & SE & $95 \% \mathrm{Cl}$ & p-value \\
\hline Improvement mRS & LDL & 0.979 & 0.012 & $(0.956-1.003)$ & 0.081 \\
\hline vs Non-improvement mRS & GOT & 0.992 & 0.006 & $(0.98 \mathrm{I}-\mathrm{I} .003)$ & 0.161 \\
\hline & $\mathrm{HbAIC}$ & 0.568 & 0.345 & $(0.289-1.1 \mid 8)$ & 0.102 \\
\hline & Coronary artery disease & 6.473 & 0.911 & $(1.085-38.629)$ & $0.04^{*}$ \\
\hline & r.CCA.RI & $3.56 * 10^{-7}$ & 6.135 & $\left(2.136 * 10^{-12}-0.059\right)$ & $0.016 *$ \\
\hline
\end{tabular}

Notes: After hospitalization mRS - Before hospitalization mRS, $\geq 0$, indicating non-improvement or stationary and $<0$ indicating improvement in patient condition; ${ }^{*} \mathrm{p}$-value $<0.05$ significant.

Abbreviations: mRS, Modified Rankin Scale; LDL, low-density lipoprotein; GOT, Glutamic Oxaloacetic Transaminase; HbAIC, hemoglobin AIc; r, right; CCA, common carotid artery; RI, resistance index; OR, odds ratio; SE, standard error; $\mathrm{Cl}$, confidence interval.

\section{Discussion}

In addition to brain stem infarct, middle cerebral artery (MCA) ischemic stroke stands out as the most precarious type, as its initial presentations often occur all of a sudden with the cerebral oedematous changing within a few days of the index episode, often requiring intensive care monitoring to prevent neurological deterioration, respiratory distress, and subsequent in-hospital infection. ${ }^{18}$ As mentioned in the introduction section, the current gold standard treatment for MCA ischemic stroke (excluding other aetiologies, including cardiogenic emboli) is for an in-charge stroke neurologist to administer intravenous thrombolytic interventions (r-tPA) and/or thrombectomy. If a stroke patient is diagnosed within the therapeutic time window of $\leq 4.5$ hours for r-tPA and $\leq 6$ hours for IA, the 3-month of functional recovery from the 
Table 5 Decision Tree Analysis of Baseline MCA Stroke Patient Datasets

\begin{tabular}{|c|c|c|c|c|c|}
\hline Dependent Variable & Variable & OR & SE & $95 \% \mathrm{Cl}$ & p-value \\
\hline \multirow{21}{*}{$\begin{array}{l}\text { Improvement mRS vs Non- } \\
\text { improvement mRS }\end{array}$} & Barthel Index & 5.637 & 0.283 & (3.237-9.8I5) & $<0.000 I^{*}$ \\
\hline & L.CCA.RI & 3.29 & 0.418 & (I.448-7.478) & $0.004 *$ \\
\hline & L.CCA.PI & 3.109 & 0.448 & $(1.292-7.478)$ & $0.011 *$ \\
\hline & R.CCA.RI & 3.011 & 0.423 & $(1.315-6.896)$ & $0.009 *$ \\
\hline & R.CCA.PI & 2.369 & 0.376 & $(1.134-4.948)$ & $0.022^{*}$ \\
\hline & T_CHO & 1.858 & 0.218 & $(1.211-2.849)$ & $0.005^{*}$ \\
\hline & $\mathrm{HbAIC}$ & 1.853 & 0.228 & $(1.185-2.897)$ & $0.007^{*}$ \\
\hline & LDL & 1.801 & 0.221 & $(1.169-2.776)$ & $0.008^{*}$ \\
\hline & R.ECA.RI & 1.67 & 0.257 & $(1.008-2.768)$ & $0.046 *$ \\
\hline & No. of comorbidities & 1.639 & 0.186 & $(1.137-2.361)$ & $0.008^{*}$ \\
\hline & WBC & 0.528 & 0.208 & $(0.352-0.793)$ & $0.002 *$ \\
\hline & Hospital days & 0.517 & 0.205 & $(0.346-0.773)$ & $0.001 *$ \\
\hline & NIHSS & $0.34 I$ & 0.210 & $(0.226-0.514)$ & $<0.000 I^{*}$ \\
\hline & CPK & 0.311 & 0.475 & $(0.123-0.790)$ & $0.014^{*}$ \\
\hline & $\mathrm{K}$ & 0.291 & 0.581 & $(0.093-0.910)$ & $0.034^{*}$ \\
\hline & GOT & 0.247 & 0.534 & $(0.087-0.705)$ & $0.009 *$ \\
\hline & R.ECA.PI & 3.693 & 0.808 & $(0.758-17.993)$ & 0.106 \\
\hline & L.ECA.RI & 2.75 & 0.592 & $(0.862-8.776)$ & 0.088 \\
\hline & Age & 2.344 & 0.497 & $(0.886-6.209)$ & 0.087 \\
\hline & Blood sugar before meal & 0.654 & 0.240 & $(0.408-1.047)$ & 0.077 \\
\hline & L.ECA.PI & 0.626 & 0.2554 & $(0.379-1.033)$ & 0.067 \\
\hline
\end{tabular}

Notes: After hospitalization mRS - Before hospitalization $\mathrm{mRS}, \geq 0$, indicating non-improvement or stationary and $<0$ indicating improvement in patient condition; *p-value $<0.05$ significant.

Abbreviations: mRS, Modified Rankin Scale; NIHSS, National Institute of Health Stroke Scale; LDL, low-density lipoprotein; GOT, Glutamic Oxaloacetic Transaminase; HbAIC, hemoglobin Alc; Sugar, sugar before meals; T_CHO, Total Cholesterol; CPK, Creatine phospho kinase; WBC, White Blood Cell; r, right side; I, left side; CCA, common carotid artery; ECA, external carotid artery; PI, perfusion Index; RI, resistance index; OR, odds ratio; SE, standard error; Cl, confidence interval.

Table 6 Decision Tree Data Analysis for Receiver Operating Curve and Cut-Off Points Searching

\begin{tabular}{|l|c|c|c|c|c|c|c|c|c|c|}
\hline \multicolumn{2}{|l|}{ Value } & $\begin{array}{c}\text { Cut-Off } \\
\text { Point }\end{array}$ & Sensitivity & I-Specificity & AUC & 95\% CI & PPV & NPV & +LR & -LR \\
\hline Outcome & Barthel Index & 57.5 & 0.686 & 0.405 & 0.675 & $(0.627-0.723)$ & 0.636 & 0.648 & 1.694 & 0.528 \\
Measurement & NIHSS & 3.5 & 0.72 & 0.491 & 0.653 & $(0.604-0.702)$ & 0.601 & 0.638 & 1.466 & 0.550 \\
\hline Blood chemicals & HbAIC & 6.15 & 0.724 & 0.586 & 0.561 & $(0.501-0.622)$ & 0.570 & 0.583 & 1.235 & 0.667 \\
& T_CHO & 164.5 & 0.663 & 0.514 & 0.573 & $(0.514-0.633)$ & 0.571 & 0.582 & 1.290 & 0.693 \\
& WBC & 7.25 & 0.608 & 0.451 & 0.578 & $(0.521-0.636)$ & 0.569 & 0.589 & 1.348 & 0.714 \\
\hline Personal & Hospital days & 6.5 & 0.77 & 0.634 & 0.571 & $(0.519-0.622)$ & 0.556 & 0.607 & 1.215 & 0.628 \\
information & No. of & 2.5 & 0.523 & 0.401 & 0.569 & $(0.518-0.62)$ & 0.573 & 0.549 & 1.304 & 0.796 \\
& comorbidities & & & & & & & & & \\
\hline Carotid duplex & r.CCA.RI & 0.785 & 0.376 & 0.324 & 0.5 & $(0.445-0.556)$ & 0.487 & 0.456 & 1.160 & 0.923 \\
\hline
\end{tabular}

Notes: The model is set to find the predicted value, and using the predicted value to run the ROC curve to seek for the most suitable segmentation probability. A predicted value less than this probability is classified as 0 , and one greater than it is classified as I, to calculate the confusion matrix in mathematical manner. The decision tree utilizes the gini index to classify variables. Its purpose is to find the cut point to divide the variables into two categories (improvement or non-improvement). Please refer this part to statistical section.

Abbreviations: NIHSS, National Institute of Health Stroke Scale; HbAIC, hemoglobin AIcT_CHO; Total Cholesterol; WBC, White Blood Cell; AUC, area under curve; $r$, right side; CCA, common carotid artery; RI, resistance index; PPV, Positive predictive value; NPV, negative predictive value; +LR, positive likelihood ratio; -LR, negative likelihood ratio; CDS, carotid duplex scan.

index stroke event is satisfactory when compared to those without treatment. However, a great number of patients remain outside of this golden standard treatment. ${ }^{19,20}$ Apart from not fitting into the scores of NIHSS and the r-tPA inclusion and exclusion criteria, the shared decision-making of family members deciding not to take thrombolytic therapy is another 
reason. This is caused by the in-charge physician explaining that the risk of cerebral bleeding after the treatment is $6 \%$, a percentage which can often cause families to decide on palliative protocols and treatments that are more conservative. Upon reviewing the currently published literature, it is obvious that a previous intake of anticoagulant medication leads to the majority of ischemic stroke patients not taking bridging therapy, as this could double the chance of cerebral or systemic bleeding risk potentially causing harm to them. ${ }^{22,23}$ Moreover, great many papers address the importance of r-tPA and/or thrombectomy treatment, seldom mentioning the outcome prediction and management of a group of patients not receiving r-tPA and/or IA treatments. In the current research, we focused on this clinical information gap by finding out the baseline contributors that might jeopardize the long-term functionality, as well as validating the role of carotid duplex parameters in terms of early favourable functional recovery. We believe that this is the first research to provide incharge clinicians with easy and uncomplicated hints from baseline datasets and clinical neuro-related imaging studies to help with their decision-making and to explain the situation to the family more clearly, as well as forecast the long-term outcome of the patient.

Table 2 reveals a rather paradoxical phenomenon whereby the improvement group has unfavourable initial NIHSS and Barthel index scores, with an elevated white blood cell count. We hypothesize that this group of individuals might have had a less fair outcome assessment (higher NIHSS score and lower points on the Barthel Index score), which could prompt in-charge clinicians to treat patients more aggressively by putting them on a more stringent "treatment protocol" to prevent collateral damage, such as: hospital infection, pneumonia, or urinary tract infection, which is why patient ultimate outcome (1-year mRS score) is better than the improvement group. In the current study, the result delineated that patients' lipid, blood sugar and previous comorbidities play significant roles in altering the patient's long-term outcome, as well as it being crucial to control the above-mentioned during the acute phase of a stroke index episode and subsequent discharge period. The other feature of the current study is to find the cut-off point for important baseline parameters for first-line doctors to differentiate favourable and unfavourable long-term outcomes beforehand. In Table 6, the results correspond to Tables 1 and 2, indicating that NIHSS, the Barthel index, lipid profile, blood sugar check-up, and white blood cell count evaluation are more precise and more important. This baseline routine documentation should be kept in mind when evaluating stroke patients. Regarding neuroimaging studies, it is a pity that we were unable to find any significant parameters through the baseline CT/CTA/CTP and cerebral magnetic resonance imaging studies, although some published papers have documented that Tmax and contralateral middle cerebral artery stenosis/occlusion might hamper the already damaged symptomatic side of index middle cerebral artery infarction that contralateral side of the basal vasculature could not savaged the symptomatic core and pending ischemic cerebral parenchyma. ${ }^{21-24}$

In terms of bio-data analysis, to be on the safe side, our research team initially utilized the conventional logistic regression method to select the important variables from the baseline datasets. However, we did not observe a strong correlation between the long-term outcome and the variables from the carotid duplex and neuroimaging parameters. We further adopted the commercially available package, CHAID decision tree, to again select the most influential predictors. To our great amazement, several detection locations for the carotid duplex (all related to PI and RI) were having a great impact on long-term functionality. Additionally, we observed several overlapping crucial variables between the two data analytical systems (Barthel Index, NIHSS, number of comorbidities, total cholesterol, HbA1c, and white blood cell count), which enabled us to use the ROC and Youden Index methodology, producing the cut-off points for each important predictor (Tables 5 and 6). The reason for this is to select the important predictors more cautiously as each analytical system has its own strengths and weaknesses and the two systems can ensure the accuracy of our results.

Table 3 correlates each underlying disease with its outcome assessment (mRS), as we use the Chi-square test of independence to gradually screen out the less important variables from the list to come up with the most important ones.

In Table 3, we use Yule's Q test method to select the most influential underlying disease that could alter a patient's long-term outcome, demonstrating dementia, chronic kidney disease, and hyperlipidaemia, as the top 3 risk factors that inversely correlate with the outcome assessment. We turned our attention to dementia, in particular, as the results of Table 1, showed that the improvement group had fewer patients with a previous diagnosis of dementia. The underlying cause could be attributed to the fact that patients with dementia might lack the willingness to move or to be selfmotivated, requiring others to help them in their daily life, therefore meaning the degree of physical recovery will ultimately be favourable compared to the group without dementia or mild cognitive impairment. This fact requires more 
case numbers and more prospective studies to prove otherwise, as the current study has only recruited a small number of patients with dementia, and it cannot be applied to the general consensus. ${ }^{25-27}$

Upon reviewing the current literature, it has been shown that RI is mostly used for the extra-cranial large arteries of the carotid and vertebral systems, whereas PI is commonly used for the assessment of intracranial blood flow velocity. ${ }^{28}$ The normal values for PI and RI are typically $<1.2$ and $<0.75$, respectively. However, to the best of our knowledge, any correlation between PI and RI values, or the magnitude of increase in values of PI and RI, remains unknown. There is no clear documentation on disease severity and the correlation of values it presents. ${ }^{28,29}$ In this study, we observed a positive correlation between the right CCA RI and patients with a long-term outcome, with the lower value presented, the more protective power and better net mRS shown (Tables 4 and 6). The explanation for this might be attributed to a lower value of the carotid system possibly generating more collateral flow to cross-irrigate the symptomatic ipsilateral stroke hemisphere to salvage the already damaged cerebral tissue in order to further restore its normal function. ${ }^{30,31}$ The downside of this current study is that we did not carry out a single recording on the right-side stroke or left-side stroke. However, based on Table 5, no matter whether it was a right-side or left-side stroke, we observed right-side and leftside CCA PI and RI both play a significant role and have a great impact on the long-term physical functionality of patients.

The strength of this study lies in the fact that patient datasets, including the baseline demographics, neuroimaging studies, and carotid duplex exams were collected and crosschecked with the $\mathrm{CCH}$ computer-based 2000 system to ensure the accuracy of the datasets. Furthermore, all of the collected datasets were analysed by the same specialist to avoid treatment methodology associated variability. The two systems of statistical analyses ensure the accuracy of the data results, avoiding the bias and influenced by other confounders. Moreover, all participants were followed up for periods in excess of a year, and outcome assessment tools were conducted by the same case manager and neurologist to ensure alignment with the dataset's accuracy and consistency. The weakness of this study is that all of the collated cases belong to the same ethnicity, therefore it is suggested that the findings are only applied to the same ethnic background, and general external application should be interpreted carefully. Subsequent rehabilitative programs and co-admixture medication drugs for patients might be confounders that could affect the data results leading to misinterpretation. The following NIHSS and Barthel Index scores are not well documented in the follow-up settings. Thus, a long-term functionality comparison regarding of neurological and physical recovery could not be made. Follow-up carotid duplex and in-hospital trans-cranial colour coded duplex datasets, which could reflect the degree of improvement and comparison and intracranial flows condition after medical treatment, are not available. The objective restoration and quantitative measurements of cerebral blood flows after the conventional treatment could

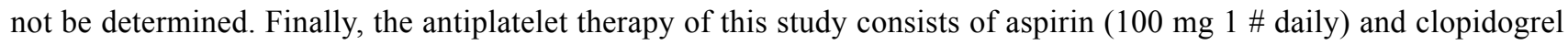
(75 mg 1 \# daily). ${ }^{32}$ We did not record patient preference nor intake and could not compare the effects of the two medications. More large-scale trials are needed to validate the current study results.

\section{Conclusion}

In the current study, long-term functional outcomes of MCA ischemic stroke patients receiving conventional medical treatment seem to correlate well with their pre-admission NIHSS, Barthel index score, total cholesterol and HbAlc levels. In addition, previously presented underlying hyperlipidaemia, chronic kidney disease, or demented state of the patients are correlated reversely with stroke patient's long-term favourable physical capability. Furthermore, the parameters derived from routinely arranged carotid duplex exam (PI and RI) might play a significant role in altering patient long term functional outcome and is more evident over common carotid artery detection. The above-mentioned information from baseline patient basic datasets could provide first-line clinicians with simple uncomplicated hints to help them with their clinical decision-making process.

\section{Data Sharing Statement}

The datasets used and/or analysed during the current study are available from the corresponding authors on reasonable request. 


\section{Ethics Approval and Consent to Participate}

The Institutional Review Board of Changhua Christian Hospital (CCH) approved this retrospective cohort study, which was performed at Changhua Christian Hospital. Informed consent was waived due to decoding between the original datasets and personal information, as well as approval from the IRB of CCH (\#201121).

\section{Consent for Publication}

Written informed consent was waived by patients for publication of this retrospective cohort study.

\section{Acknowledgments}

Our team would like to thank Miss Yi-Chun Tu, Ting-Wei Jiang, Chia-Chen Fan, Ya-Li Chen for data sorting and technical support, and Mr Tim Stubbings for his English proofreading.

\section{Author Contributions}

All authors made a significant contribution to the work reported, whether that is in the conception, study design, execution, acquisition of data, analysis and interpretation, or in all these areas; took part in drafting, revising or critically reviewing the article; gave final approval of the version to be published; have agreed on the journal to which the article has been submitted; and agree to be accountable for all aspects of the work.

\section{Funding}

No funding was secured for this study.

\section{Disclosure}

The authors declare that they have no competing interests.

\section{References}

1. Ha SH, Chang JY, Lee SH, et al. Mechanism of stroke according to the severity and location of atherosclerotic middle cerebral artery disease. J Stroke Cerebrovasc Dis. 2021;30(2):105503. doi:10.1016/j.jstrokecerebrovasdis.2020.105503

2. Treadwell SD, Thanvi B. Malignant middle cerebral artery (MCA) infarction: pathophysiology, diagnosis and management. Postgrad Med J. 2010;86(1014):235-242. doi:10.1136/pgmj.2009.094292

3. Chiang T, Messing RO, Chou WH. Mouse model of middle cerebral artery occlusion. J Vis Exp. 2011;48:2761.

4. Powers WJ, Rabinstein AA, Ackerson T, et al. Guidelines for the early management of patients with acute ischemic stroke: 2019 update to the 2018 guidelines for the early management of acute ischemic stroke: a guideline for healthcare professionals from the American Heart Association/ American Stroke Association. Stroke. 2019;50(12):e344-e418. doi:10.1161/STR.0000000000000211

5. Winship IR. Cerebral collaterals and collateral therapeutics for acute ischemic stroke. Microcirculation. 2015;22(3):228-236. doi:10.1111/ micc. 12177

6. Walcott BP, Miller JC, Kwon CS, et al. Outcomes in severe middle cerebral artery ischemic stroke. Neurocrit Care. 2014;21(1):20-26. doi:10.1007/ s12028-013-9838-x

7. Xu W, Zhang X, Chen H, Zhao Z, Zhu M. Prevalence and outcome of young stroke patients with middle cerebral artery stenosis. BMC Neurol. 2021;21(1):99. doi:10.1186/s12883-021-02125-8

8. Hwang DY, Matouk CC, Sheth KN. Management of the malignant middle cerebral artery syndrome. Semin Neurol. 2013;33(5):448-455. doi:10.1055/s-0033-1364211

9. Godoy D, Piñero G, Cruz-Flores S, Alcalá Cerra G, Rabinstein A. Malignant hemispheric infarction of the middle cerebral artery. Diagnostic considerations and treatment options. Neurologia. 2016;31(5):332-343. doi:10.1016/j.nrl.2013.02.009

10. Parenti N, Bastiani L, Palazzi C, Urrea K, Silingardi M. Could Duplex carotid ultrasound be useful in the assessment of volume status and fluid responsiveness? Eur J Intern Med. 2020;76:110-114. doi:10.1016/j.ejim.2020.02.006

11. Murray CSG, Nahar T, Kalashyan H, Becher H, Nanda NC. Ultrasound assessment of carotid arteries: current concepts, methodologies, diagnostic criteria, and technological advancements. Echocardiography. 2018;35(12):2079-2091. doi:10.1111/echo.14197

12. Serena J. Ultrasonography of the progression of atherosclerotic plaques. Rev Neurol. 1999;29(9):851-856. PMID: 10696661.

13. Lin CM, Liu CK, Chang YJ, Chen WL, Lu HH. Reversed ophthalmic artery flow following ischemic stroke: a possible predictor of outcomes following carotid artery stenting. Neurol Res. 2019;41(2):132-138. doi:10.1080/01616412.2018.1544744

14. Nana P, Spanos K, Antoniou G, et al. The effect of carotid revascularization on the ophthalmic artery flow: systematic review and meta-analysis. Int Angiol. 2021;40(1):23-28. doi:10.23736/S0392-9590.20.04448-X

15. Reynolds PS, Greenberg JP, Lien LM, Meads DC, Myers LG, Tegeler CH. Ophthalmic artery flow direction on color flow duplex imaging is highly specific for severe carotid stenosis. J Neuroimaging. 2002;12(1):5-8. doi:10.1111/j.1552-6569.2002.tb00082.x

16. Lipsitz SR, Fitzmaurice G. An extension of Yule's Q to multivariate binary data. Biometrics. 1994;50(3):847-852. PMID: 7981405. doi:10.2307/ 2532798 
17. Song YY, Lu Y. Decision tree methods: applications for classification and prediction. Shanghai Arch Psychiatry. 2015;27(2):130-135. doi:10.11919/j.issn.1002-0829.215044

18. Shanbhag NC, Henning RH, Schilling L. Long-term survival in permanent middle cerebral artery occlusion: a model of malignant stroke in rats. Sci Rep. 2016;6:28401. doi:10.1038/srep28401

19. Kang BS, Kwon HM, Ryu WS, Lee YS. Prognosis of symptomatic and asymptomatic middle cerebral artery occlusion. Cerebrovasc Dis. $2008 ; 26$ (5):489-493. doi:10.1159/000155986

20. Guanci MM. Management of the patient with malignant hemispheric stroke. Crit Care Nurs Clin North Am. 2020;32(1):51-66. doi:10.1016/j. cnc.2019.11.003

21. Trillo S, Ramos MC, Aguirre C, et al. Assessment of collateral circulation using perfusion CT in middle cerebral artery thrombectomy-treated patients. J Stroke Cerebrovasc Dis. 2020;29(7):104805. doi:10.1016/j.jstrokecerebrovasdis.2020.104805

22. Sundseth J, Sundseth A, Jacobsen EA, et al. Predictors of early in-hospital death after decompressive craniectomy in swollen middle cerebral artery infarction. Acta Neurochir. 2017;159(2):301-306. doi:10.1007/s00701-016-3049-0

23. Albert AF, Kirkman MA. Clinical and radiological predictors of malignant middle cerebral artery infarction development and outcomes. $J$ Stroke Cerebrovasc Dis. 2017;26(11):2671-2679. doi:10.1016/j.jstrokecerebrovasdis.2017.06.041

24. Köhrmann M, Schwab S. Hemicraniectomy for malignant middle cerebral artery infarction. Curr Opin Crit Care. 2009;15(2):125-130. doi:10.1097/MCC.0b013e328321826e

25. Kalaria RN, Akinyemi R, Ihara M. Stroke injury, cognitive impairment and vascular dementia. Biochim Biophys Acta. 2016;1862(5):915-925. doi:10.1016/j.bbadis.2016.01.015

26. Hachinski V. The convergence of stroke and dementia. Arq Neuropsiquiatr. 2018;76(12):849-852. doi:10.1590/0004-282x20180148

27. Pendlebury ST, Rothwell PM. Prevalence, incidence, and factors associated with pre-stroke and post-stroke dementia: a systematic review and meta-analysis. Lancet Neurol. 2009;8(11):1006-1018. doi:10.1016/S1474-4422(09)70236-4

28. Bellner J, Romner B, Reinstrup P, et al. Transcranial Doppler sonography pulsatility index (PI) reflects intracranial pressure (ICP). Surg Neurol. 2004;62:45-51. doi:10.1016/j.surneu.2003.12.007

29. Yazici B, Erdogmus B, Tugay A. Cerebral blood flow measurements of the extracranial carotid and vertebral arteries with Doppler ultrasonography in healthy adults. Diagn Interv Radiol. 2005;11:195-198.

30. Huttner HB, Schwab S. Malignant middle cerebral artery infarction: clinical characteristics, treatment strategies, and future perspectives. Lancet Neurol. 2009;8(10):949-958. doi:10.1016/S1474-4422(09)70224-8

31. Schiemanck SK, Post MW, Witkamp TD, Kappelle LJ, Prevo AJ. Relationship between ischemic lesion volume and functional status in the 2nd week after middle cerebral artery stroke. Neurorehabil Neural Repair. 2005;19(2):133-138. doi:10.1177/154596830501900207

32. Pereira NL, Rihal CS, So DYF, et al. Clopidogrel Pharmacogenetics. Circ Cardiovasc Interv. 2019;12(4):e007811. doi:10.1161/ CIRCINTERVENTIONS.119.007811

\section{Publish your work in this journal}

Neuropsychiatric Disease and Treatment is an international, peer-reviewed journal of clinical therapeutics and pharmacology focusing on concise rapid reporting of clinical or pre-clinical studies on a range of neuropsychiatric and neurological disorders. This journal is indexed on PubMed Central, the 'PsycINFO' database and CAS, and is the official journal of The International Neuropsychiatric Association (INA). The manuscript management system is completely online and includes a very quick and fair peer-review system, which is all easy to use. Visit http://www.dovepress.com/testimonials.php to read real quotes from published authors.

Submit your manuscript here: https://www.dovepress.com/neuropsychiatric-disease-and-treatment-journal 